\title{
GENUS 2 SEMI-REGULAR COVERINGS WITH LIFTING SYMMETRIES
}

\author{
YOLANDA FUERTES* \\ Departamento de Matemáticas, U. Autonoma de Madrid, 28049 Madrid, Spain \\ e-mail: yolanda.fuertes@uam.es \\ and ALEXANDER MEDNYKH** \\ Sobolev Institute of Mathematics, Novosibirsk State University, 630090, Novosibirsk, Russia \\ e-mail:smedn@mail.ru
}

(Received 23 June 2006; revised 22 October 2007; accepted 27 March 2008)

\begin{abstract}
In this paper, we obtain algebraic equations for all genus 2 compact Riemann surfaces that admit a semi-regular (or uniform) covering of the Riemann sphere with more than two lifting symmetries. By a lifting symmetry, we mean an automorphism of the target surface which can be lifted to the covering. We restrict ourselves to the genus 2 surfaces in order to make computations easier and to make possible to find their algebraic equations as well. At the same time, the main ingredient (Main Proposition) depends neither on the genus, nor on the order of the group of lifting symmetries. Because of this, the paper can be thought as a generalisation for the non-normal case to the question of lifting automorphisms of a compact Riemann surface to a normal covering, treated, for instance, by E. Bujalance and M. Conder in a joint paper, or by P. Turbek solely.
\end{abstract}

2000 Mathematics Subject Classification. 30F10, 14H30, 14 H45.

1. Introduction. A compact Riemann surface in this paper is a closed (orientable) one-dimensional complex manifold. There are a few different, but essentially equivalent approaches to investigate compact Riemann surfaces. The first one comes from the classical paper by Hurwitz [12]. Here, a Riemann surface has been defined as a branched covering of the extended complex plane (the Riemann sphere) with prescribed branching data. More precisely, an $n$-fold covering is defined by the Hurwitz system, consisting of a finite number of critical points $z_{1}, z_{2}, \ldots, z_{r}$ and an $r$-tuple $\left(\xi_{1}, \xi_{2}, \ldots, \xi_{r}\right)$ of substitutions of $S_{n}$ satisfying the equation $\xi_{1} \xi_{2} \cdots \xi_{r}=1$. The corresponding covering surface is connected if and only if the group $\left\langle\xi_{1}, \xi_{2}, \ldots, \xi_{r}\right\rangle$ generated by $\xi_{1}, \xi_{2}, \ldots, \xi_{r}$ is a transitive subgroup of $S_{n}$. If $\xi_{j}=\left(1^{s_{1}} 2^{s_{2}} \cdots n^{s_{n}}\right), j=1,2, \ldots, r$ consists of $s_{k}^{j}$ cycles of length $k$, then the pre-image of the point $z_{j}$ has exactly $k$ branch points of order $s_{k}^{j}$. Since any Riemann surface has a non-constant meromorphic function, both definitions are equivalent. From the beginning, a Riemann surface was considered as a set of definition of a multi-valued analytical function. This gives a way to define a Riemann surface as an algebraic equation $R(x, y)=0$, where $R$ is a complex polynomial. Again, by [7], we have an equivalent definition. The equations for Riemann surfaces of genus $\leq 5$

*Partially supported by Grants MTM2006-01859 and MTM2006-14688.

** Partially supported by RFBR (06-01-00153) and Fondecyt (1060378). 
with prescribed automorphism group were obtained in $[\mathbf{4}, \mathbf{1 8}-\mathbf{2 0}, \mathbf{2 4}]$. One more and a very fruitful approach was developed by A. M. Macbeath, D. Singerman, G. Jones, E. Bujalance and others. They considered a Riemann surface as a factor space $\mathbb{H} / \Gamma$, where $\Gamma$ is a Fuchsian group acting by isometries on the hyperbolic plane $\mathbb{H}$. By the Poincaré, Klein and Köbe uniformisation theorems, we again obtain an equivalent definition.

In the present paper, we investigate the problem of the relationship between the Hurwitz and Algebraic equation definitions. Our aim is to obtain algebraic equations for Riemann surfaces of a small genus arising as a branched covering of the Riemann sphere. To do this, we will widely use the theory of Fuchsian groups and the orbifold language. For basic definitions, we refer the reader to the book [7]. In the case of regular coverings, the solution of the problem is well known and presented in papers $[4,6,8,9,19,24,28]$ and many others. To find algebraic equations for irregular coverings is still open and a very difficult problem. Some partial results in this direction are obtained in [3] and [16]. We restrict ourself to the case of semi-regular coverings. By definition, we suppose that the substitutions $\xi_{1}, \xi_{2}, \ldots, \xi_{r}$ in the Hurwitz system are regular, that is, each $\xi_{j}=\left(\ell_{j}^{m_{j}}\right)$ consists of $m_{j}$ cycles on length $\ell_{j}$ with $\ell_{j} m_{j}=n, j=$ $1,2, \ldots, r$. We will refer to the signature $\left(\ell_{1}, \ell_{2}, \ldots, \ell_{r}\right)$ as the branching data of the semi-regular covering. All regular coverings are necessary semi-regular, the converse is not true. See, for example [8] and [25].

In this paper, we consider only the case of genus 2 semi-regular coverings of the Riemann sphere. Even under such restriction, there are 30 admissible branching data $\left(\ell_{1}, \ell_{2}, \ldots, \ell_{r}\right)$ for $r=3,4,5,6$ obtained in [1]. Given branching data produce a few non-equivalent coverings of a prescribed genus. In particular, it follows from the GAP calculation done by A. Hulpke [11] that there are 39 non-equivalent genus 2 semiregular coverings with branching data $(2,2,2,3)$, and only three of them are regular. There are also 22 admissible branching data for $r=3$. In this case, by J. Neubücher, the number of respective coverings is highly large. Summarising, one should say that the problem of finding algebraic equations for all genus 2 semi-regular coverings of the sphere is quite complicated. To make this problem more realistic, we put one more condition for coverings under consideration, namely, to have at least three lifting symmetries. (See the next section for more detailed definition.) In this case, we are able to produce the full list of genus 2 covering surfaces. It is given in Theorem 3 . This is the main result of the paper.

In spite the general problem seems to be difficult, we provide a certain background (Main Lemma and Main Proposition) to develop it in the future.

Our interest in the subject was motivated by $\mathrm{H}$. Farkas question about an algebraic equation for the genus 2 Riemann surface admitting a semi-regular (but non-regular) five-fold covering of the Riemann sphere branched over three points. This question was posed to the second named author during his stay in Jerusalem in 1999, and indeed, initiated us to write the present paper.

It follows from [21] and [25] that there are four non-equivalent genus 2 coverings of the Riemann sphere with branching data $(5,5,5)$. Three of them are regular and produce the same Riemann surface $y^{5}=x(x-1)$ and the last one is irregular. The corresponding covering surface as well as a positive answer to the Farkas question are given by the curve from Theorem 3 defined by the equation

$$
F_{5}: y^{2}=x^{6}+\frac{118}{5} x^{3}+1
$$


2. Preliminaries results. Let us give some notation and elementary facts about regular (or normal) and semi-regular (or uniform) coverings in the framework of Fuchsian groups. By a semi-regular covering we mean one in which every point at the fiber of a branch point has the same ramification index for every branch point of the covering (see for example [17]). This definition extends the definition of a uniform covering, that is, a semi-regular covering of the Riemann sphere with at most three critical values (see for example [27]), and we may use this notation as well. We will refer to a covering as a regular (or normal) one if the group of covering transformations acts transitively on the fiber of any point. Let $f: X \rightarrow Y$ be a covering. An automorphism $h^{\prime}$ of the target surface $Y$ is called a lifting symmetry of $f$ if there is an automorphism $h$ of $X$ such that $f \circ h=h^{\prime} \circ f$. We note that the trivial automorphism of $Y$ lifts to the group of covering transformations of $f$, while a lift of a non-trivial one does not belong to the covering group.

EXAMPLE. Let $X$ be the genus 2 Riemann surface defined by the equation $y^{5}=$ $x(x-1)$ and $f: X \rightarrow \mathbb{C P}^{1}$ is the regular covering such that $f(x, y)=x$. Then, $f$ admits only one non-trivial lifting symmetry $h^{\prime}(x)=1-x$. The five possible lifts $h$ of $h^{\prime}$ to the surface $X$ are given by the following equations:

$$
h=h_{k}(x, y)=(1-x, \exp ((2 k-1) \pi \sqrt{-1} / 5) y), \quad k=1,2,3,4,5 .
$$

Due to the Uniformization theorem, an arbitrary compact Riemann surface $X$ of genus $g \geq 2$ can be viewed as a quotient space $X \cong \mathbb{H} / K$, where $\mathbb{N}$ is the upper half-plane and $K$ is the uniformising group. $K$ is a discrete subgroup of $P S L_{2}(\mathbb{R})$, the group of conformal automorphisms of $\mathbb{H}$, isomorphic to the fundamental group of the surface (so acting fixed point freely on $\mathbb{H}$, or torsion free), very often termed as a (Fuchsian) surface group. From elementary facts of covering space theory, every

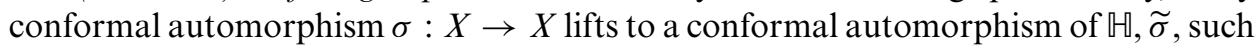
that $\tilde{\sigma} K \widetilde{\sigma}^{-1}=K$, i.e: $\widetilde{\sigma}$ belongs to the normalizer of $K$ in $P S L_{2}(\mathbb{R})$, let us denote it from now on by $N(K)$, and vice versa. With the analogous notation, let us now consider a compact Riemann surface of genus $g \geq 2, X \cong \mathbb{H} / K$, admitting an automorphism group isomorphic to $G$ with quotient orbifold $X / G \cong Y \cong \mathbb{H} / \Gamma$ (that is, $\Gamma=\langle K, \widetilde{G}\rangle$, where $\widetilde{G}$ is a lift of the group $G$ to $\mathbb{M})$. The latter is, obviously, equivalent to the existence of a surface kernel epimorphism $\varphi: \Gamma \rightarrow G$ (that is, $\operatorname{Ker}(\varphi)$ is a surface group) and $K=\operatorname{Ker}(\varphi)$. We will say that two surface kernel epimorphisms are equivalent if they differ by post-composition with an automorphism of $G$.

The signature $\left[\gamma ; m_{1}, m_{2}, \ldots, m_{r}\right]$ of the Fuchsian group $\Gamma$ encodes its presentation as an abstract group, i.e:

$$
\begin{array}{ll}
\text { generators: } & a_{1}, b_{1}, \ldots, a_{\gamma}, b_{\gamma} ; \quad x_{1}, x_{2}, \ldots, x_{r} \\
\text { relations : } & x_{1}^{m_{1}}=\cdots=x_{r}^{m_{r}}=\prod_{i=1}^{\gamma}\left[a_{i}, b_{i}\right] x_{1} x_{2} \cdots x_{r}=1,
\end{array}
$$

where $\left[a_{i}, b_{i}\right]=a_{i} b_{i} a_{i}^{-1} b_{i}^{-1}$; as well as the geometric nature of the action of $G: \gamma$ is the genus of the quotient surface $Y$ and the $\left\{m_{i}\right\}$ are the branching orders of the covering $X \rightarrow Y$ (for more details, see [10]). From now on, we will refer, not only for a normal covering but also for a semi-regular one, to the collection of natural numbers $\left(\gamma ; m_{1}, \ldots, m_{r}\right)$ as the branching data of the covering. Moreover, the area of a fundamental region for $\Gamma$, a Fuchsian group with the above signature, is $2 \pi \mu(\Gamma)$, 
where

$$
\mu(\Gamma)=2(\gamma-1)+\sum_{i=1}^{r}\left(1-\frac{1}{m_{i}}\right) .
$$

It is well known that for any inclusion of Fuchsian groups $K \leq \Gamma$, one has

$$
[\Gamma: K]=\mu(K) / \mu(\Gamma) .
$$

From now on, we will refer to $K \unlhd \Gamma$ as the normal inclusion of Fuchsian groups that realizes the normal or regular covering $X \rightarrow Y=X / G$ and to $G$ as the covering group. On the other hand, we will use the standard notation $\left[m_{1}, m_{2}, \ldots, m_{r}\right]$ when $\gamma=0$.

Let $f: X \rightarrow Y$ be a semi-regular covering of degree $d$ ramified over $r$ points $\left\{y_{j}^{\prime}\right\}_{j=1}^{r}$ with ramification index $m_{j}$, for every $j=1, \ldots, r$. Let us denote the restriction of $f$ to the associated $d$-sheeted unramified covering by $f: \stackrel{\circ}{X} \rightarrow \stackrel{\circ}{Y}$; that is, $\stackrel{\circ}{Y}=Y-\left\{y_{j}^{\prime}\right\}_{j=1}^{r}$ and $\stackrel{\circ}{X}=f^{-1}(\stackrel{\circ}{Y})$. We denote the fundamental group of $\stackrel{\circ}{Y}$ at any non-critical point of $f, y \in \stackrel{\circ}{Y}$, by $\pi(\stackrel{\circ}{Y} ; y)$. As $\pi(\stackrel{\circ}{Y} ; y)$ has the following presentation as an abstract group

$$
\begin{aligned}
\text { generators : } & \alpha_{1}, \beta_{1}, \ldots, \alpha_{\gamma}, \beta_{\gamma} ; \quad v_{1}, v_{2}, \ldots, v_{r} \\
\text { relations : } & \prod_{i=1}^{i=\gamma}\left[\alpha_{i}, \beta_{i}\right] v_{1} v_{2} \cdots v_{r}=1,
\end{aligned}
$$

$\gamma$ being the genus of $Y$, there is an obvious epimorphism $\rho$ from $\pi(\stackrel{\circ}{Y} ; y)$ to a Fuchsian group $\Gamma$ with signature $\left[\gamma ; m_{1}, m_{2}, \ldots, m_{r}\right]$. The above considerations have the following implications. Consider the monodromy representation of the covering $f, \quad m_{f}: \pi(\stackrel{\circ}{Y} ; y) \rightarrow S_{d}$, where $S_{d}$ is the permutation group on $d$ letters, and the homomorphism $m: \Gamma \rightarrow S_{d}$ is given by $m \circ \rho=m_{f}$. Then, we have an inclusion of Fuchsian groups $K \leq \Gamma$ ( $K$ being the pre-image by $m$ of a point stabilizer in the monodromy group of $\left.f, M_{f}:=\operatorname{Im}\left(m_{f}\right)=\operatorname{Im}(m) \leq S_{d}\right)$ that realizes the covering in the sense that there exist isomorphisms of compact Riemann surfaces $\psi: X \rightarrow \mathbb{W} / K$ and $\xi: Y^{\prime} \rightarrow \mathbb{H} / \Gamma$ such that $\xi \circ f=p \circ \psi$, where $p: \mathbb{H} / K \rightarrow \mathbb{H} / \Gamma$ is the natural projection induced by $K \leq \Gamma$. Let us point it out that $K$ is a Fuchsian surface group because the condition that the covering is semi-regular implies that $K$ is torsion free (see [14]).

Let us observe that $\operatorname{Ker}(m)=\cap_{i=1}^{d} \gamma_{i} K \gamma_{i}^{-1},\left\{\gamma_{1}=1, \ldots, \gamma_{d}\right\}$ being a set of representatives of the quotient $\Gamma / K$ on the left. Therefore, if $f$ is a normal covering, we have that $\operatorname{Ker}(m)=K$ and $M_{f}$ is isomorphic to the group of covering transformations or, in other words, to the covering group.

REMARK 1. For the well-known equivalence between compact Riemann surfaces or algebraic curves and their algebraic function fields, morphisms $f: X \rightarrow Y$ correspond to function field extensions of degree equal to the degree of the morphisms, that is, $f^{*}(\mathbb{C}(Y)) \hookrightarrow \mathbb{C}(X)$ and $\left[\mathbb{C}(X): f^{*}(\mathbb{C}(Y))\right]=\operatorname{deg}(\mathrm{f})$. In particular, normal coverings with covering group $G$ correspond to Galois function field extensions and, of course, the function field of the quotient surface is the fixed field under the action of the Galois group of the extension; namely, $\mathbb{C}(Y) \cong(\mathbb{C}(X))^{G}$. For instance, we refer to [7] for basic and general background about algebraic curves or compact Riemann surfaces and their morphisms or surjective holomorphic maps.

Now we have to state some necessary background about semi-regular coverings in the setting of Fuchsian groups. We need to study the question when an automorphism 
$h^{\prime}$ of the target surface $Y$ lifts to a semi-regular covering $f: X \rightarrow Y$, that is, when there exists $h \in \operatorname{Aut}(X)$ such that $f \circ h=h^{\prime} \circ f$.

\section{Lifting automorphisms.}

Main lemma. Let $K, \Gamma$ and $\Lambda$ be Fuchsian groups. Assume that $K$ is a torsion free subgroup of index $d$ in $\Gamma$ and $\Gamma \unlhd \Lambda$. Then, for any $\lambda$ belonging to $\Lambda$, the automorphism induced by $\lambda$ on $\mathbb{H} / \Gamma$ lifts to an automorphism of $\mathbb{H} / K$ if and only if there exists an element of $S_{d}, \tau$, such that $\phi \circ \chi_{\lambda}=\chi_{\tau} \circ \phi$, where $\chi_{\lambda}$ and $\chi_{\tau}$ are the automorphisms of $\Gamma$ and $S_{d}$ defined by conjugation under $\lambda$ and $\tau$, respectively, and $\phi: \Gamma \rightarrow S_{d}$ is the permutation representation of $\Gamma$ obtained acting on a set of left coset representatives of $\Gamma / K$.

Proof. First of all, let us consider a set $\left\{\gamma_{1}=i d, \ldots, \gamma_{d}\right\}$ of representatives of the quotient $\Gamma / K$ on the left. If we denote the image of $\phi$ in $S_{d}$ as the monodromy group $M_{\phi}$; for every $i=1, \ldots, d, K_{i}=\gamma_{i} K \gamma_{i}^{-1}$ where, of course, if $K:=K_{1}=\phi^{-1}(\mathrm{Stab}(1))$, then one has that $\operatorname{Ker}(\phi)=\cap_{1}^{d} K_{i}$ and $M_{\phi} \cong \Gamma /\left(\cap_{1}^{d} K_{i}\right)$.

On the other hand, for any $\lambda \in \Lambda$, as $\Lambda$ is contained in the normalizer of $\Gamma$ in $P S L_{2}(\mathbb{R}), \chi_{\lambda}$ is an automorphism of $\Gamma$; therefore, $\chi_{\lambda}: \Gamma \rightarrow \Gamma$ induces an automorphism of the monodromy group $\Gamma /\left(\cap_{1}^{d} K_{i}\right)$, if and only if $\chi_{\lambda}\left(\cap_{1}^{d} K_{i}\right)=\cap_{1}^{d} K_{i}$. If we start with this assumption, then we have that $\cap_{i=1}^{d} K_{i}=\chi_{\lambda}\left(\cap_{1}^{d} K_{i}\right)=\lambda\left(\cap_{1}^{d} K_{i}\right) \lambda^{-1}=\cap_{1}^{d}\left(\lambda K_{i} \lambda^{-1}\right)$. Therefore, we must have $\lambda\left(K_{i}\right) \lambda^{-1}=K_{j_{i}}$, for every $i=1, \ldots, d$. In particular, $\gamma_{j_{1}}^{-1} \circ \lambda$ belongs to the normalizer of $K_{1}=K$, which finishes this part of the proof.

Finally, in order to prove the necessary condition, let us denote by $\left\{\gamma_{i}^{\prime}\right\}_{i=1}^{d}$ the elements of $\Gamma$ such that $\lambda \circ \gamma_{i}=\gamma_{i}^{\prime} \circ \lambda$. Then, as it was reminded in Section $2, \lambda$ induces an automorphism on the space of $K$-orbits of $\mathbb{W}$ if and only if $\lambda K \lambda^{-1}=K=K_{1}$. Therefore,

$$
\begin{aligned}
\chi_{\lambda}\left(\cap_{1}^{d} K_{i}\right) & =\lambda\left(\cap_{1}^{d} K_{i}\right) \lambda^{-1}=\cap_{1}^{d}\left(\lambda K_{i} \lambda^{-1}\right) \\
& =\cap_{1}^{d}\left(\lambda \gamma_{i} K \gamma_{i}^{-1} \lambda^{-1}\right) \\
& =\cap_{1}^{d}\left(\gamma_{i}^{\prime} \lambda K \lambda^{-1}\left(\gamma_{i}^{\prime}\right)^{-1}=\cap_{1}^{d}\left(\gamma_{i}^{\prime} K\left(\gamma_{i}^{\prime}\right)^{-1}\right)=\cap_{1}^{d} K_{i}\right.
\end{aligned}
$$

The last equalities follow from the fact that $\left\{\gamma_{i}^{\prime}=\gamma_{j_{i}} \circ k_{i}\right\}_{i=1}^{d}$ constitutes another set of representatives of the quotient $\Gamma / K$ on the left and $k_{i} \in K$, for every $i=1, \ldots, d$. Consequently, as $\lambda$ belongs to $\Lambda$ and $\Lambda$ is contained in the normalizer of $\Gamma$ in $P S L_{2}(\mathbb{R})$, one has that $\chi_{\lambda}$ induces an automorphism on the monodromy group $M_{\phi}$, by conjugation under $\tau=\left(j_{1} \cdots j_{d}\right)$, that makes the diagram commutative in the Main Lemma.

It is more interesting to state the result in the setting of semi-regular coverings of compact Riemann surfaces but, first of all, we need to make the following observation. For every automorphism of $Y, h^{\prime}$, that lifts to the semi-regular covering $f: X \rightarrow Y$ corresponding to the inclusion of Fuchsian groups $K \leq \Gamma$ with $\Gamma$ of signature $\left[\gamma ; m_{1}, \ldots, m_{r}\right]$, we must have that $h^{\prime}$ preserves the branch points of $f$ on $Y$, that is, if we consider the set of branch points of $f$ on $Y$ that partitions into sets $\Sigma_{l}$, for $l=1, \ldots, k$, each one with $s_{l}$ points of the same branch order and $\sum_{l=1}^{k} s_{l}=r$, then $h^{\prime}\left(\Sigma_{l}\right)=\Sigma_{l}$. Moreover, using an argument similar to the one explained in 
[22, pp. 499-500], we obtain that $h^{\prime}$ lifts to a conformal self-homeomorphism $\widetilde{h^{\prime}}: \mathbb{U} \rightarrow \mathbb{U}$ and $\widetilde{h^{\prime}} \Gamma{\widetilde{h^{\prime}}}^{-1}=\Gamma$; therefore, $\widetilde{h^{\prime}} \in N(\Gamma)$ in $P S L_{2}(\mathbb{R})$.

MaIN PROPOSITION. Let $f: X \rightarrow Y$ be a semi-regular covering of compact Riemann surfaces of degree $d$ realised by inclusion of Fuchsian groups $K \leq \Gamma$, and $h^{\prime}$ an automorphism of $Y \cong \mathbb{I} / \Gamma$. Then, $h^{\prime}$ lifts to an automorphism $h$ of $X \cong \mathbb{U} / K$, that is, $f \circ h=h^{\prime} \circ f$ if and only if $h^{\prime}$ preserves the branch points of $f$ on $Y$ and there exists an element of $S_{d}, \tau$, such that $\phi \circ \chi_{\widetilde{h}^{\prime}}=\chi_{\tau} \circ \phi$, where $\chi_{\widetilde{h}^{\prime}}$ and $\chi_{\tau}$ are the automorphism of $\Gamma$ and $S_{d}$ defined by conjugation by $\widetilde{h}^{\prime} \in P S L_{2}(\mathbb{R})$ and $\tau$, respectively, and $\phi: \Gamma \rightarrow S_{d}$ is the permutation representation of $\Gamma$ obtained by its action on a set of left coset representatives of $\Gamma / K$.

Proof. It follows from the above observation and the result of the Main Lemma.

REMARK 2. The above results show that in order to find every semi-regular covering $f: X \rightarrow Y$ with group of automorphisms $H^{\prime}$ of $Y$ that lifts to a group of automorphisms $H$ of $X$, we must have a surface group $K$ satisfying the following diagram of Fuchsian groups, where $\Lambda=\left\langle\Gamma, \cup_{h^{\prime} \in H^{\prime}} \widetilde{h^{\prime}}\right\rangle$ :

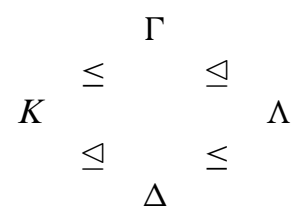

The above diagram of inclusions of Fuchsian groups corresponds to the following diagram of coverings of compact Riemann surfaces



4. Statement of the result. Let $f: X \rightarrow Y$ be a semi-regular covering, where $X$ is a genus 2 Riemann surface. Then, the genus of the Riemann surface $Y$ is two, one or zero. According to [1], there are 34 possible branching data for the covering $f$. If $f$ is regular, only 20 of them remain [5] ([1, List 2]). In this case, the full list of covering groups as well as algebraic equations for the Riemann surfaces $X$ are known for a long time since Bolza's paper [4]. It was already mentioned in Section 1 that semi-regular coverings are regular, but the converse is not true. We call a semi-regular covering to be proper if it is not regular. Still, by [1], we have 14 (or even more) possible branching data for proper semi-regular coverings.

From now on, we restrict ourself to proper semi-regular coverings having at least three lifting symmetries. The following theorem shows that there are only five Riemann surfaces with the above property. All of them are coverings of the Riemann sphere branched over three points, that is, they are Belyi surfaces [15]. 
THEOREM 3. The genus 2 compact Riemann surfaces admitting a proper semi-regular covering with at least three lifting symmetries are given by the following equations:

$$
\begin{aligned}
& C_{1}: y^{2}=x^{6}+\left(\frac{-164 \sqrt{2}-178}{27}\right) x^{3}+1, \\
& C_{2}: y^{2}=x^{6}+\left(\frac{164 \sqrt{2}-178}{27}\right) x^{3}+1, \\
& C_{3}: y^{2}=x^{6}+(2214 \sqrt{2}-3130) x^{3}+1, \\
& C_{4}: y^{2}=x^{6}+(-2214 \sqrt{2}-3130) x^{3}+1, \\
& F_{5}: y^{2}=x^{6}+\frac{118}{5} x^{3}+1 .
\end{aligned}
$$

The last covering has branching data $(5,5,5)$, while the other four have branching data $(4,4,4)$. For each covering, the group of lifting symmetries is isomorphic to $\mathbb{D}_{3}$. The whole automorphism group of the surfaces under consideration is isomorphic to $\mathbb{D}_{6}$.

5. Proof of the Theorem. Before starting with the proof of the theorem, we summarize the procedure we are going to follow.

First we look at all possible genus 2 semi-regular coverings $f: X \rightarrow Y$ such that $Y$ possesses a non-trivial automorphism group $H^{\prime}$ satisfying the necessary conditions to lift to an automorphism group of $X$ given in Remark 2 (Lemma 6). We use Broughton's paper [5] and the lattice of conmensurability of two-generator Fuchsian groups of Maclachlan and Rosenberger [23] in order to find such coverings. Then, using the Main Lemma, we prove that for all these coverings, the group $H^{\prime}$ does actually lift to an automorphism group $H$ of $X$ (Proposition 7).

It turns out that the quotient of $X$ under $H$ is always the Riemann sphere with three branch points. Finally, using the cross-ratio and suitable Belyi functions on the Riemann sphere, we obtain their algebraic equations (Propositions 8 and 9).

In [5], one can find all possible signatures for the groups $\Delta$ in the above diagram for our genus 2 case. The signatures for the group $\Gamma$ in the same diagram are given in [11, List 2]. Recall that we are looking only to those coverings whose group $H^{\prime}=\Lambda / \Gamma$ of lifting symmetries contains at least three elements. Then, its lift $H=\Delta / K$ also contains at least three elements. We are not going to study the case of one- and twofold coverings $X \rightarrow Y$, since they are regular. Then, we have the following restriction on the group inclusions: the indices of subgroups $|\Gamma: K|,|\Delta: K|,|\Lambda: \Gamma|,|\Lambda: \Delta| \geq 3$. In particular, we obtain $|\Lambda: K| \geq 9$. Hence, by [11, List 2], the group $\Lambda$ has either signature $[2,2,2,3]$ or is an arithmetic triangle group.

Let $\Lambda$ be a group of signature $[2,2,2,3]$. We have $\Gamma \unlhd \Lambda$ and $|\Lambda: \Gamma||\Gamma: K|=\mid \Lambda$ : $K \mid=12$. Hence, the index $|\Lambda: \Gamma|$ is equal to 3 or 4 . We note that $\Lambda=[2,2,2,3]$ has no normal subgroups of index 3. Therefore, $|\Lambda: \Gamma|=4$. Since $\Lambda$ has no elements of order 4 , we obtain $\Lambda / \Gamma \cong \mathbb{Z}_{2} \oplus \mathbb{Z}_{2}$. Up to equivalency, there is only one epimorphism $\Lambda$ onto $\mathbb{Z}_{2} \oplus \mathbb{Z}_{2}$, with kernel $\Gamma=[3,3,3,3]$. Hence, $\Gamma=[3,3,3,3]$ is the unique normal subgroup of index 4 in $\Lambda$. By assumption, $K \leq \Gamma$, where $K$ is the genus 2 surface group. Then, $X=\mathbb{U} / K \rightarrow \mathbb{U} / \Gamma$ is a three-fold covering of the Riemann sphere with branching data $(3,3,3,3)$. By [25], it is always regular. This is not the case under consideration. 
So, the group $\Lambda$ is an arithmetic triangle group. On the other hand, it follows from [5] that, apart from Fuchsian groups with signature [2, 2, 2, 2, 2], the groups $\Delta$ have to be triangle or quadrangle groups with signatures that appear in the lattices of commensurability classes obtained in the paper of C. Maclachlan and G. Rosenberger [23]. Therefore, it is enough to check there in order to get all possible diagrams as required in Remark 2.

In what it concerns to $[2,2,2,2,2]$, after inspection, we found the inclusions given in Lemma 4 below. To obtain the possible candidates, we use a simple area argument. Then we use Theorem 1 in D. Singerman's paper [26], in order to check the inclusions that really occur:

LEMMA 4. The possible inclusions of a Fuchsian group $\Delta_{i}$ that has signature [2, 2, 2, 2, 2] inside a triangle group $\Lambda_{i}$ are the following:
(i) $\Gamma_{1}=[2,2,2,2,2]_{1} \leq[2,3,8]=\Lambda_{1}$ with index 12
(ii) $\Gamma_{2}=[2,2,2,2,2]_{2} \leq[2,3,9]=\Lambda_{2}$ with index 9
(iii) $\Gamma_{3}=[2,2,2,2,2]_{3} \leq[2,3,12]=\Lambda_{3}$ with index 6
(iv) $\Gamma_{4}=[2,2,2,2,2]_{4} \leq[2,4,6]=\Lambda_{4}$ with index 6
(v) $\Gamma_{5}=[2,2,2,2,2]_{5} \unlhd[2,4,8]=\Lambda_{5}$ with index 4
(vi) $\Gamma_{6}=[2,2,2,2,2]_{6} \leq[2,4,5]=\Lambda_{6}$ with index 10 .

Proof. The proof that those triangle groups are the only possible Fuchsian groups that contain a quadrangle group with signature $[2,2,2,2,2]$ can be done using a simple area argument as exposed in Section 1. Moreover, according to [26, Theorem 1], the existence of the above inclusions of Fuchsian groups comes from the existence of the following permutation representations $\theta_{i}$ of the action of the generators of the corresponding triangle group $\Lambda_{i}$, on a set of left coset representatives of $\Lambda_{i} / \Delta_{i}$, for $i=1,2,3,4,5$ and 6; namely:

$$
\begin{array}{rlrl}
\theta_{1}:[2,3,8] \rightarrow S_{12} & \theta_{2}:[2,3,9] \rightarrow S_{9} \\
& x_{1} \rightarrow(1,6)(2,10)(3,7)(5,11)(8,9)(4)(12) & x_{2} \rightarrow(1)(2)(4)(6)(7)(3,9)(5,8) \\
y_{1} \rightarrow(1,4,7)(2,6,11)(3,10,8)(5,9,12) & y_{2} \rightarrow(1,3,2)(4,9,5)(6,8,7) \\
z_{1} \rightarrow(1,2,3,4)(5,6,7,8)(9,10,11,12) & z_{2} \rightarrow(1,2,3,4,5,6,7,8,9) \\
& & \\
\theta_{3}:[2,3,12] \rightarrow S_{6} & \theta_{4}:[2,4,6] \rightarrow S_{6} \\
& x_{3} \rightarrow(1,4)(2)(3)(5)(6) & x_{4} \rightarrow(1)(2)(3)(5)(4,6) \\
y_{3} \rightarrow(1,6,5)(2,4,3) & y_{4} \rightarrow(1,4,3,2)(5,6) \\
z_{3} \rightarrow(1,2,3,4,5,6) & z_{4} \rightarrow(1,2,3,4,5,6) \\
& & \\
\theta_{5}:[2,4,8] \rightarrow S_{4} & \theta_{6}:[2,4,5] \rightarrow S_{10} \\
x_{5} \rightarrow(1)(2)(3)(4) & x_{6} \rightarrow(1)(2)(6)(7)(3,8)(4,10)(5,9) \\
y_{5} \rightarrow(1,4,3,2) & y_{6} \rightarrow(1,9,3,2)(4,8,7,6)(5,10) \\
z_{5} \rightarrow(1,2,3,4) & z_{6} \rightarrow(1,2,3,4,5)(6,7,8,9,10) .
\end{array}
$$

In what follows, we will also need the following lemma.

LEMMA 5. Consider the finite group $\mathbb{D}_{6}=\left\langle a, b: a^{6}=b^{2}=(a b)^{2}=1\right\rangle$, then for any Fuchsian group $\Delta$ with signature [2, 2, 2, 3], there exist three non-equivalent surface 
kernel epimorphisms $\varphi_{i}: \Delta \rightarrow \mathbb{D}_{6}$ given by

$$
\begin{array}{lll}
\varphi_{1}(x)=b a^{2} & \varphi_{2}(x)=b a^{5} & \varphi_{3}(x)=a^{3} \\
\varphi_{1}(y)=a^{3} & \varphi_{2}(y)=b & \varphi_{3}(y)=b a^{5} \\
\varphi_{1}(z)=b a^{3} & \varphi_{2}(z)=a^{3} & \varphi_{3}(z)=b \\
\varphi_{1}(t)=a^{2} & \varphi_{2}(t)=a^{2} & \varphi_{3}(t)=a^{2} .
\end{array}
$$

Proof. We will use the character table of $\mathbb{D}_{6}$ in order to get the number of different surface kernel epimorphisms $\varphi$ from a Fuchsian quadrangle group $\Delta=\langle x, y, z, t|$ $\left.x^{2}=y^{2}=z^{2}=t^{3}=x y z t=1\right\rangle$ with signature $[2,2,2,3]$ to the group $\mathbb{D}_{6}$ :

$\begin{array}{ccccccc}C & 1 A & 2 A_{1} & 2 A_{2} & 2 C & 3 B & 6 A \\ |C| & 1 & 3 & 3 & 1 & 2 & 2 \\ & \text { id } & b & a b & a^{3} & a^{2} & a \\ \psi_{1} & 1 & 1 & 1 & 1 & 1 & 1 \\ \psi_{2} & 1 & -1 & -1 & 1 & 1 & 1 \\ \psi_{3} & 1 & 1 & -1 & -1 & 1 & -1 \\ \psi_{4} & 1 & -1 & 1 & -1 & 1 & -1 \\ \psi_{5} & 2 & 0 & 0 & -2 & -1 & 1 \\ \psi_{6} & 2 & 0 & 0 & 2 & -1 & 1 .\end{array}$

Let us observe, first of all, that any epimorphism $\varphi$ has to apply one of the three order two generators of $\Delta$ to the central involution $a^{3}$ and all of them to elements belonging to different conjugacy classes because otherwise, composing $\varphi$ with the quotient epimorphism $\pi: \mathbb{D}_{6} \rightarrow \mathbb{D}_{6} / Z\left(\mathbb{D}_{6}\right)$, where $Z\left(\mathbb{D}_{6}\right)=\left\langle a^{3}\right\rangle$ denotes the center of $\mathbb{D}_{6}$, one gets a contradiction with the fact that we must have $\pi \circ \varphi(x y z t)=\pi \circ \varphi(1)=1$. Thus, the number of different epimorphisms equals the number of solutions of the equations $\bar{x}_{1}^{2}=\bar{x}_{2}^{2}=\bar{x}_{3}^{2}=\bar{x}_{4}^{3}=\bar{x}_{1} \bar{x}_{2} \bar{x}_{3} \bar{x}_{4}=1$, where $\bar{x}_{1}, \bar{x}_{2}$ and $\bar{x}_{3}$ belong to any permutation of the conjugacy classes $2 A_{1}, 2 A_{2}$ and $2 C$, and $\bar{x}_{4}$ belongs to $3 B$. This number $n_{\varphi}$ can be easily calculated by using the well-known formula in [13],

$$
n_{\varphi}:=6 \frac{\left|2 A_{1}\right|\left|2 A_{2}\right||2 C||3 B|}{\left|\mathbb{D}_{6}\right|} \sum_{i=1}^{6} \frac{\psi_{i}\left(\bar{x}_{1}\right) \psi_{i}\left(\bar{x}_{2}\right) \psi_{i}\left(\bar{x}_{3}\right) \psi_{i}\left(\bar{x}_{4}\right)}{\psi_{i}(1)^{2}}=36
$$

Of course to obtain the number of different surface kernels, we have to take into account that two chosen image quadruples lead to the same surface kernel if and only if they differ by an automorphism of the group $\mathbb{D}_{6}$. The automorphism group of $\mathbb{D}_{6}$ has order $12=6 \times 2$ because it is, obviously, isomorphic to $\mathbb{Z}_{6} \times \mathbb{Z}_{2}$. Therefore, we obtain the total number of different kernels inside the [2, 2, 2, 3] quadrangle group to be $n_{\varphi} / 12=3$. It is easy to check that the three non-equivalent epimorphisms can be given as was stated in Lemma 5.

Let $\Lambda$ be an arithmetic triangle group. Then, the only two diagrams, as described in Remark 2, obtained after inspection in the lattices of commensurability in [23] for the genus 2 Fuchsian surface groups are as follows. 
LEMMA 6. For any $l=1,2,3,4$ and 5

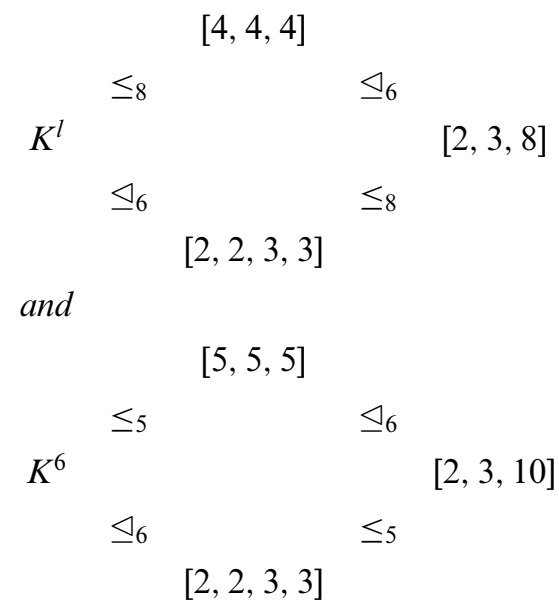

Proof. The normal inclusions that we have not already proved follow from the fact that for any natural number $N \geq 4$, there is always an embedding $\psi$ of the Fuchsian triangle group $[N, N, N]=\left\langle x, y, z: x^{N}=y^{N}=z^{N}=x y z=1\right\rangle$ inside the triangle group with signature $[2,3,2 N]=\left\langle\delta, \eta, v: \delta^{2}=\eta^{3}=v^{2 N}=\delta \eta v=1\right\rangle$ given by $\psi(x)=v^{2}, \psi(y)=\eta v^{2} \eta^{-1}, \psi(z)=\eta^{2} v^{2} \eta^{-2}$ and the quotient group of [2, 3, 2N] under $[N, N, N]$ is isomorphic to $\mathbb{D}_{3}$.

The remaining inclusions of the right-hand side of the diagrams occur because of [26, Theorem 1], and the following homomorphisms of groups:

$$
\begin{aligned}
& \theta_{1}:[2,3,8] \rightarrow S_{4} \\
& \theta_{2}:[2,2,2,3] \rightarrow S_{2} \\
& \theta_{3}:[2,3,10] \rightarrow S_{5} \\
& x \rightarrow(1)(2)(3,4) \\
& x \rightarrow(1)(2) \\
& x \rightarrow(1,2)(3)(4,5) \\
& y \rightarrow(4)(1,3,2) \\
& y \rightarrow(1,2) \\
& y \rightarrow(1,4,3)(2)(5) \\
& z \rightarrow(1,2,3,4) \\
& z \rightarrow(1,2) \\
& z \rightarrow(1,2,3,4,5) \text {. }
\end{aligned}
$$

Let us observe that from $\theta_{1}$, we obtain a Fuchsian group of signature $[2,2,2,3]$ inside the triangle group [2, 3, 8]; from $\theta_{2}$, a Fuchsian group of signature $[2,2,3,3]$ inside another one with signature [2, 2, 2, 3], and finally, from $\theta_{3}$, a Fuchsian group of signature $[2,2,3,3]$ inside the triangle group $[2,3,10]$. where

The groups $K^{l}$ referred to in the statement of our lemma arise as $K^{l}=\phi_{l}^{-1}(\mathrm{~S} t a b(1))$

$$
\begin{aligned}
& \phi_{1}:[4,4,4] \rightarrow S_{8} \\
& x \rightarrow(1,8,5,3)(2,7,4,6) \\
& \phi_{2}:[4,4,4] \rightarrow S_{8} \\
& y \rightarrow(1,7,4,5)(2,3,6,8) \\
& x \rightarrow(1,6,8,5)(2,7,3,4) \\
& z \rightarrow(1,2,3,4)(5,6,7,8) \\
& y \rightarrow(1,3,4,7)(2,5,6,8) \\
& z \rightarrow(1,2,3,4)(5,6,7,8) \text {. } \\
& \phi_{3}:[4,4,4] \rightarrow S_{8} \\
& x \rightarrow(1,8,3,5)(2,6,7,4) \\
& \phi_{4}:[4,4,4] \rightarrow S_{8} \\
& y \rightarrow(1,7,2,5)(3,4,8,6) \\
& x \rightarrow(1,8,5,7)(2,3,6,4) \\
& z \rightarrow(1,2,3,4)(5,6,7,8) \\
& y \rightarrow(1,6,8,5)(2,7,3,4) \\
& z \rightarrow(1,2,3,4)(5,6,7,8) \text {. }
\end{aligned}
$$




$$
\begin{aligned}
\phi_{5}:[4,4,4] & \rightarrow S_{8} & \phi_{6}:[5,5,5] & \rightarrow S_{5} \\
x & \rightarrow(1,6,3,8)(2,5,4,7) & x & \rightarrow(1,2,3,4,5) \\
y & \rightarrow(1,5,3,7)(2,8,4,6) & y & \rightarrow(1,2,4,5,3) \\
z & \rightarrow(1,2,3,4)(5,6,7,8) & & \rightarrow(1,4,5,2,3) .
\end{aligned}
$$

This is a necessary condition that turns out to be sufficient. To see this, one has to use the Main Lemma. The existence of the corresponding group homomorphisms follows from the next proposition.

Proposition 7. There exist five compact Riemann surfaces of genus 2 , $\mathbb{H} / K^{l}$ for $l=1,2,3,4,5$, having a semi-regular covering with branching data $(4,4,4)$. These are the natural projections induced by the inclusion of groups $\phi_{l}: \mathbb{H} / K^{l} \rightarrow \mathbb{H} /[4,4,4]$ for $l=1,2,3,4,5$. There is in addition a proper semi-regular covering $\mathbb{H} / K^{6} \rightarrow \mathbb{H} /[5,5,5]$ with branching data $(5,5,5)$, where $K^{6} \leq[5,5,5]$. In all cases, the respective group of lifting symmetries is isomorphic to $\mathbb{D}_{3}$.

Proof. As we have seen in the previous proof, if we denote $[2,3,8]=\langle\delta, \eta, v$ : $\left.\delta^{2}=\eta^{3}=v^{8}=\delta \eta v=1\right\rangle$, then $[4,4,4]=\left\langle x, y, z: x^{4}=y^{4}=z^{4}=x y z=1\right\rangle$, where $x=v^{2}, y=\eta v^{2} \eta^{-1}$ and $z=\eta^{2} v^{2} \eta^{-2}$. On the other hand, let us observe that

$$
\chi_{\delta}(x)=\delta x \delta^{-1}=y, \quad \chi_{\delta}(y)=\delta y \delta^{-1}=x \quad \text { and } \quad \chi_{\delta}(z)=\delta z \delta^{-1}=x^{-1} y^{-1} .
$$

Then, if we consider the permutations in $S_{8}$ given by $\tau_{1}=(1,2)(3,8)(5,6), \tau_{1}^{\prime}=$ $(2,8,7)(3,5,4), \tau_{2}=(3,6)(4,8)(5,7), \tau_{2}^{\prime}=(1,2,7)(5,8,6), \quad \tau_{3}=(2,3)(4,6)(7,8)$, $\tau_{3}^{\prime}=(1,4,7)(3,6,5), \quad \tau_{4}=(1,3)(2,5)(4,8), \quad \tau_{4}^{\prime}=(1,6,7)(2,4,3), \quad \tau_{5}=(1,2)(3,4)$ $(6,8)$ and $\tau_{5}^{\prime}=(2,6,5)(4,8,7)$, we have

$$
\phi_{i} \circ \chi_{\delta}=\chi_{\tau_{i}} \circ \phi_{i}, \quad \phi_{i} \circ \chi_{\eta}=\chi_{\tau_{i}^{\prime}} \circ \phi_{i},
$$

for $i=1,2,3,4$ and 5 .

Analogously, if we consider $[2,3,10]=\left\langle\delta, \eta, v: \delta^{2}=\eta^{3}=v^{10}=\delta \eta v=1\right\rangle$, then $[5,5,5]=\left\langle x, y, z: x^{5}=y^{5}=z^{5}=x y z=1\right\rangle$, where $x=v^{2}, y=\eta v^{2} \eta^{-1}$ and $z=$ $\eta^{2} v^{2} \eta^{-2}$. On the other hand, if we denote by $\tau=(1,4)(2,5) \in S_{5}$ and $\tau^{\prime}=(1,3,2) \in$ $S_{5}$, then

$$
\phi_{6} \circ \chi_{\delta}=\chi_{\tau} \circ \phi_{6}
$$

and

$$
\phi_{6} \circ \chi_{\eta}=\chi_{\tau^{\prime}} \circ \phi_{6} .
$$

Finally, let us observe that $\mathbb{H} / K^{5} \rightarrow \mathbb{H} /[4,4,4]$ is a regular covering with covering group isomorphic to

$$
Q_{8}=\left\langle a, b: a^{4}=b^{4}=1, a^{2}=b^{2}, b a b^{-1}=a^{-1}\right\rangle .
$$

As all elements in the group $[2,3,8] /[4,4,4] \cong \mathbb{D}_{3}$ lift to automorphisms of $\mathbb{H} / K^{5}$, this compact Riemann surface admits a group of automorphisms of order, at least, 48 . According to Broughton in [5], this is, precisely, its order and the group is isomorphic to $G L_{2}(3)$, the linear group of $2 \times 2$ matrices with coefficients in the field of three elements. 
In order to obtain the algebraic curves uniformized by these genus 2 Fuchsian surface groups, we observe that the genus 2 compact Riemann surfaces admitting $\mathbb{D}_{3}$ as a group of automorphisms admit as well a group of automorphisms isomorphic to $\mathbb{D}_{6}$. We also note that these Riemann surfaces belong to a one-complex parameter family $\mathcal{F}$, with the following algebraic hyperelliptic model (see [4]):

$$
S_{t} \equiv\left\{y^{2}=\left(x^{3}-d^{3}\right)\left(x^{3}-1 / d^{3}\right)=x^{6}-t x^{3}+1\right\} \in \mathcal{F},
$$

for every $t \in \mathbb{C}-\{ \pm 2\}$ and $t=d^{3}+1 / d^{3}$; the generators of the automorphism group being given on each surface $S_{t} \in \mathcal{F}$ by

$$
a(x, y)=\left(\xi_{3} x,-y\right) \quad \text { and } \quad b(x, y)=\left(1 / x, y / x^{3}\right), \quad \text { with } \xi_{3}=\exp (2 \pi \sqrt{-1} / 3) .
$$

Let us observe that $a^{3}(x, y)=(x,-y)$ is the hyperelliptic involution.

PROPOSITION 8. The genus 2 algebraic curves admitting a semi-regular covering of the Riemann sphere with branching data $(4,4,4)$ are given by the following equations:

$$
\begin{aligned}
& C_{1}: y^{2}=x^{6}+\left(-\frac{164 \sqrt{2}-178}{27}\right) x^{3}+1, \\
& C_{2}: y^{2}=x^{6}+\left(\frac{164 \sqrt{2}-178}{27}\right) x^{3}+1, \\
& C_{3}: y^{2}=x^{6}+(2214 \sqrt{2}-3130) x^{3}+1, \\
& C_{4}: y^{2}=x^{6}+(-2214 \sqrt{2}-3130) x^{3}+1, \\
& C_{5}: y^{2}=x\left(x^{4}-1\right) .
\end{aligned}
$$

The last covering is regular, while the other four are proper semi-regular. In all cases, the respective group of lifting symmetries is isomorphic to $\mathbb{D}_{3}$.

Proof. It follows from the last paragraph in the proof of Proposition 7 that $C_{5} \cong$ $\mathbb{H} / K^{5}$ has automorphism group isomorphic to $G L_{2}(3)$. Such a curve was already obtained in [4].

The hyperelliptic involution does not belong to the automorphism subgroup constituted by lifting the elements of the quotient group $[2,3,8] /[4,4,4] \cong \mathbb{D}_{3}$. Therefore, an invariant meromorphic function under a subgroup of the automorphism group isomorphic to $\mathbb{D}_{3}$ is given by

$$
f(x, y)=x^{3}+y+\frac{-x^{3}-y+1+t}{x^{3}+y+1} .
$$

This function is obtained as composition of

$$
(x, y) \mapsto\left(x^{3}, y\right) \in\left\{\hat{y}^{2}=\hat{x}^{2}-t \hat{x}+1\right\},
$$

whose image is a singular model of the Riemann sphere, followed by the inverse of the parametrization of this singular model given by $z=\hat{x}+\hat{y}$, and finally, followed by

$$
z \mapsto z+\frac{-z+1+t}{z+1}
$$


The first morphism is equivalent to taking the quotient by the subgroup $\left\langle a^{2}\right\rangle$, which is a normal subgroup of $\left\langle a^{2}, b a^{3}\right\rangle \cong \mathbb{D}_{3}$; therefore, $\left\langle b a^{3}\right\rangle$ projects to an order 2 subgroup of the automorphism group of the quotient surface $\left\langle\overline{b a^{3}}\right\rangle$, and the last morphism corresponds to taking the quotient by the conjugate under the inverse of the parametrization written above of this cyclic subgroup. We get the following images of the fixed points under $f$ :

$$
\begin{aligned}
P_{1}:=(-1, \sqrt{t+2}) & \longmapsto-2+2 \sqrt{t+2}=Q_{1}, \\
P_{2}:=(-1,-\sqrt{t+2}) & \longmapsto-2-2 \sqrt{t+2}=Q_{2}, \\
P_{3}:=(0,1) & \longmapsto 1+\frac{t}{2}=Q_{3}, \\
P_{4}:=\infty_{1} & \longmapsto \infty=Q_{4} .
\end{aligned}
$$

Here, $P_{4}$ is one of the two fixed points of $a^{2}$ on the same orbit under the action of $\left\langle a^{2}, b a^{3}\right\rangle ; P_{3}$ is one of the other two fixed points of $a^{2}$, of course, on the same orbit under the action of $\left\langle a^{2}, b a^{3}\right\rangle ; P_{2}$ is one of the two fixed points of $b a^{3}$, and finally, $P_{1}$ is the other fixed point of $b a^{3}$.

On the other hand, let us observe that this Riemann surface admits a semi-regular meromorphic function $\psi_{l}: \mathbb{H} / K^{l} \rightarrow \mathbb{H} / \Lambda$ with three critical values $\left\{q_{k}\right\}_{k=1}^{3}$, so it only remains to obtain the function field extension (up to field isomorphisms) corresponding to the natural quotient $\mathbb{H} / \Delta \rightarrow \mathbb{H} / \Lambda$, that is, the field extension $\mathbb{C}\left(h_{j}(z)\right) \hookrightarrow \mathbb{C}(z)$ corresponding to the meromorphic function $h_{j}: \mathbb{C P}^{1} \rightarrow \mathbb{C P}^{1}$ (see Remark 1). In order to do that, we strongly use the conditions imposed by the fact that $h_{j} \circ f$ has to be a semi-regular covering with given ramification indexes. This last consideration gives us a set of positive numbers $\left(\mu_{1}^{1}=3, \mu_{2}^{1}=3, \mu_{3}^{1}=1, \mu_{4}^{1}=1 ; \mu_{1}^{2}=2, \mu_{2}^{2}=2, \mu_{3}^{2}=\right.$ $\left.2, \mu_{4}^{2}=1, \mu_{5}^{2}=1 ; \mu_{1}^{3}=8\right)$, with $\left\{\mu_{n}^{k}\right\}$ being the ramification order of the points on the fiber of $q_{k}$ by $h_{j}$; and the meromorphic functions are $h_{1}(z)=\left(z^{2}+\frac{81+54 \sqrt{2}}{16}\right)\left(z^{2}-\right.$ $\left.2 z+\frac{41+22 \sqrt{2}}{16}\right)^{3}$ and $h_{2}(z)=\left(z^{2}+\frac{81-54 \sqrt{2}}{16}\right)\left(z^{2}-2 z+\frac{41-22 \sqrt{2}}{16}\right)^{3}$ (see [2]). Now, we can identify (up to composition with a Möbius transformation) the critical values of $f$, that we have already denoted by $Q_{1}, Q_{2}, Q_{3}$ and $Q_{4}$, with the corresponding points on the fiber of $q_{k}$ by $h_{j}$; let us denote them by $R_{1}^{1}=\frac{7+2 \sqrt{-10-7 \sqrt{2}}}{4}, \quad R_{2}^{1}=\frac{7-2 \sqrt{-10-7 \sqrt{2}}}{4}$, $R_{3}^{1}=\frac{3 \sqrt{-3}+3 \sqrt{-6}}{4}$ and $R_{4}^{1}=\frac{-3 \sqrt{-3}-3 \sqrt{-6}}{4}$ for $j=1$ and $R_{1}^{2}=\frac{7+2 \sqrt{-10+7 \sqrt{2}}}{4}, \quad R_{2}^{2}=$ $\frac{7-2 \sqrt{-10+7 \sqrt{2}}}{4}, R_{3}^{2}=\frac{-3 \sqrt{-3}+3 \sqrt{-6}}{4}$ and $R_{4}^{2}=\frac{3 \sqrt{-3}-3 \sqrt{-6}}{4}$ for $j=2$. Finally, we obtain the values of $t$ imposing that they have the same cross-ratio

$$
\begin{aligned}
& \lambda\left(Q_{1}, Q_{2}, Q_{3}, Q_{4}\right)=\frac{4 \sqrt{t+2}}{3+\frac{t}{2}+2 \sqrt{t+2}}=\lambda\left(R_{1}^{1}, R_{2}^{1}, R_{3}^{1}, R_{4}^{1}\right) \\
& \quad=\frac{10587+6970 \sqrt{2}+1002 \sqrt{3} \sqrt{10+7 \sqrt{2}}+756 \sqrt{6} \sqrt{10+7 \sqrt{2}}}{6(126 \sqrt{6} \sqrt{10+7 \sqrt{2}}+167 \sqrt{3} \sqrt{10+7 \sqrt{2}}+738 \sqrt{2}+1044)},
\end{aligned}
$$

that gives us $t=\frac{-164 \sqrt{2}-178}{27}$ and $t=2214 \sqrt{2}-3130$. On the other hand,

$$
\begin{aligned}
\lambda & \left(Q_{1}, Q_{2}, Q_{3}, Q_{4}\right)=\lambda\left(R_{1}^{2}, R_{2}^{2}, R_{3}^{2}, R_{4}^{2}\right) \\
& =\frac{10587-6970 \sqrt{2}-1002 \sqrt{3} \sqrt{10-7 \sqrt{2}}+756 \sqrt{6} \sqrt{10-7 \sqrt{2}}}{6(126 \sqrt{6} \sqrt{10-7 \sqrt{2}}-167 \sqrt{3} \sqrt{10-7 \sqrt{2}}-738 \sqrt{2}+1044)},
\end{aligned}
$$

that gives us $t=\frac{164 \sqrt{2}-178}{27}$ and $t=-2214 \sqrt{2}-3130$. 
We note that the above arguments are not enough for obtaining the unique curve admitting a proper semi-regular covering with branching data $(5,5,5)$. In this case, we will follow another approach.

PROPOSITION 9. The genus 2 algebraic curve admitting a proper semi-regular covering of the Riemann sphere with branching data $(5,5,5)$ is given by the equation

$$
F_{5}: y^{2}=x^{6}+\frac{118}{5} x^{3}+1
$$

The respective group of lifting symmetries is isomorphic to $\mathbb{D}_{3}$.

Proof (Following an idea by Manfred Streit). The curve $F_{5} \cong \mathbb{H} / K^{6}$ admits a semiregular covering $f_{1}$ to $\mathbb{C P}^{1}$ with branching data $(5,5,5)$, and there exists a regular meromorphic function $h_{1}: \mathbb{C P}^{1} \rightarrow \mathbb{C P}^{1}$ given by $h_{1}(x)=\left(\frac{x^{3}-1}{x^{3}+1}\right)^{2}$ that realizes the inclusion of Fuchsian groups $[5,5,5] \unlhd[2,3,10]$ with quotient group isomorphic to $\mathbb{D}_{3}$ (for details, see [15]).

On the other hand, $F_{5}$ has a normal covering $f_{2}$, with covering group isomorphic to $\mathbb{D}_{3}$ and branching data $(2,2,3,3)$. Now, by [2], the meromorphic function $h_{2}: \mathbb{C P}^{1} \rightarrow$ $\mathbb{C P}^{1}$, given by $h_{2}(y)=\frac{1}{64} y\left(3 y^{2}-10 y+15\right)^{2}$, realizes the inclusion of Fuchsian groups $[2,2,3,3] \leq[2,3,10]$. Using the same notation as in Proposition 8 , it can be equipped with the following collection of natural numbers: $\left(\mu_{1}^{1}=2, \mu_{2}^{1}=2, \mu_{3}^{1}=1 ; \mu_{1}^{2}=\right.$ $3, \mu_{2}^{2}=1, \mu_{3}^{2}=1 ; \mu_{1}^{3}=5$ ), where $\mu_{j}^{k}$ are the ramification orders of the points on the fiber of $q_{k}$ by $h_{2}$.

In terms of function field extensions, and having in mind that on $F_{5}$, we have that $h_{1} \circ f_{1}=h_{2} \circ f_{2}$, the above two paragraphs have the following translation:

$$
\begin{aligned}
& E=\mathbb{C}\left(\frac{\left(x^{3}-1\right)^{2}}{\left(x^{3}+1\right)^{2}}\right) \stackrel{6}{\hookrightarrow} \mathbb{C}(x) \stackrel{5}{\hookrightarrow} \mathbb{C}\left(F_{5}\right) ; \\
& E=\mathbb{C}\left(y\left(3 y^{2}-10 y+15\right)^{2}\right) \stackrel{5}{\hookrightarrow} \mathbb{C}(y) \stackrel{6}{\hookrightarrow} \mathbb{C}\left(F_{5}\right) .
\end{aligned}
$$

As an elementary exercise of field extensions, it follows from the fact that the greatest common divisor of 5 and 6 equals 1 , that $E=\mathbb{C}(x) \cap \mathbb{C}(y)$ and that $[\mathbb{C}(x, y): E]=5 \cdot 6$; therefore, $\mathbb{C}\left(F_{5}\right) \cong \mathbb{C}(x, y)$. We recall that on $F_{5}$, one has $h_{1} \circ f_{1}=h_{2} \circ f_{2}$; therefore, $F_{5}$ must satisfy the following algebraic equation:

$$
\left(\frac{x^{3}-1}{x^{3}+1}\right)^{2}=\frac{y\left(3 y^{2}-10 y+15\right)^{2}}{64} .
$$

By substituting $y=v^{2}$, the latter curve can be rewritten in the form $\frac{x^{3}-1}{x^{3}+1}=$ $\frac{v\left(3 v^{4}-10 v^{2}+15\right)}{8}$. Finally, by the birational transformation

$$
X=\frac{v-1}{v+1} x, \quad Y=\frac{6 \sqrt{-15}\left(8-3 v^{2}\right)}{5\left(8+9 v+3 v^{2}\right)}
$$

we obtain

$$
F_{5}: Y^{2}=X^{6}+\frac{118}{5} X^{3}+1
$$


ACKNOWLEDGEMENTS. The authors are thankful to the referee for remarks and suggestions.

\section{REFERENCES}

1. M. T. Abu-Osman and G. Rosenberger, Embedding property of surface groups, Bull. Malaysian Math. Soc. 3(2) (1980), 21-27.

2. J. Bétréma and A. Zvonkin, Plane trees and Shabat polynomials. Proceedings of the 5 th conference on formal power series and algebraic combinatorics (Florence, 1993). Discrete Math. 153(1-3) (1996), 47-58.

3. P. Boalch, Some explicit solutions to the Riemann-Hilbert problem. http://arxiv.org/ pdf/math.DG/0501464.pdf.

4. O. Bolza, On binary sextics with linear transformations into themselves, Amer. J. Math. 10 (1888), 47-60.

5. S. A. Broughton, Classifying finite group actions on surfaces of low genus, J. Pure Appl. Algebra 69 (1990), 233-270.

6. E. Bujalance and M. Conder, On cyclic groups of automorphisms of Riemann surfaces, J. London Math. Soc. 59(2) (1999), 573-584.

7. H. Farkas and I. Kra, Riemann surfaces, Graduate Texts in Math. 71 (Springer-Verlag, New York, 1980).

8. Y. Fuertes and M. Streit, Genus 3 normal coverings of the Riemann sphere branched over 4 points, Rev. Mat. Iberoamericana 22(2) (2006), 413-454.

9. G. González-Diez, Loci of curves which are prime Galois coverings of $\mathbb{C P}^{1}$, Proc. London Math. Soc. 62(3) (1991), 469-489.

10. W. H. Harvey, On branch loci in Teichmüller space, Trans. Amer. Math. Soc. 153 (1971), 387-399.

11. A. Hulpke, T. Kuusalo, M. Naatanen and G. Rosenberger, On orbifold coverings by genus 2 surfaces, Scientia, Series A: Math. Sci. 11 (2005), 45- 55.

12. A. Hurwitz, Über Riemannăsche Flachen mit gegebenen Verzweigungpunkten, Math. Ann. 39 (1891), 1-61.

13. G. A. Jones, Characters and surfaces: A survey. The atlas of finite groups: Ten years on (Birmingham, 1995), 90-118, London Math. Soc., Lecture Note Series 249 (Cambridge Univ. Press, Cambridge, UK, 1998).

14. G. Jones and D. Singerman, Theory of maps on orientable surfaces, Proc. London Math. Soc. 37(3) (1978), 273-307.

15. G. Jones and D. Singerman, Belyi functions, hypermaps and Galois groups, Bull. London Math. Soc. 28 (1996), 561-590.

16. V. E. Kruglov, Structure of the partial indices of the Riemann problem with permutationtype matrices, Math. Notes 35(2) (1984), 89-93.

17. R. S. Kulkarni, Riemann surfaces admitting large automorphism groups. Extremal Riemann surfaces (San Francisco, CA, 1995), Contemporary Math. 201, Amer. Math. Soc., Providence, RI (1997), 63-79.

18. A. Kuribayashi and H. Kimura, Automorphism groups of compact Rieman surfaces of genus five, J. Algebra 134 (1990), 80-103.

19. A. Kuribayashi and K. Komiya, On Weierstrass points and automorphisms of curves of genus three, in Algebraic Geometry (Proc. Summer Meeting, Univ. Copenhagen, Copenhagen, 1978), 253-299, Lecture Notes Math. 732 (Springer-Verlag, Berlin, Germany, 1979).

20. I. Kuribayashi and A. Kuribayashi, Automorphism groups of compact Riemann surfaces of genera three and four, J. Pure Appl. Algebra 65 (1990), 277-292.

21. J. H. Kwak and A. Mednykh, Enumeration of branched coverings of closed orientable surfaces whose branch orders coincide with multiplicity, Studia Sci. Math. Hungarica 44(2) (2007), 215-223.

22. C. Maclachahn and W. H. Harvey, On mapping-class groups and Teichmüller space, Proc. London Math. Soc. 30(3) (1975), 496-512.

23. C. Maclachlan and G. Rosenberger, Commensurability classes of two-generators Fuchsian groups. Discrete groups and geometry (Birmingham, 1991), 171-189, London Math. Soc. Lecture Note Series 173, (Cambridge Univ. Press, Cambridge, UK, 1992). 
24. K. Magaard, T. Shaska, S. Shpectorov and H. Völklein, The locus of curves with prescribed automorphism group. Communications in arithmetic fundamental groups (Kyoto, 1999/2001), Sürikaisekikenkyüsho Kökyüroku No. 1267 (2002), 112-141.

25. A. D. Mednykh, On branched coverings of Riemann surfaces with the trivial group of covering transformations, Sov. Math. Dokl. 18 (1977), 1156-1158 [translation from Dokl. Akad. Nauk SSSR 235 (1977), 1267-1269].

26. D. Singerman, Subgroups of Fuchsian groups and finite permutation groups, Bull. London Math. Soc. 2 (1970), 319-323.

27. D. Singerman and R. I. Syddall, Belyi uniformization of elliptic curves, Bull. London Math. Soc. 29 (1997), 443-451.

28. P. Turbek, A necessary and sufficient condition for lifting the hyperelliptic involution, Proc. Amer. Math. Soc. 125(9) (1997), 2615-2625. 